\title{
Management and outcomes of childhood Goodpasture's disease
}

\author{
Carmela P. Menzi ${ }^{1}$, Barbara S. Bucher ${ }^{1}$, Mario G. Bianchetti ${ }^{2}$, Gianluigi Ardissino ${ }^{3}$ and Giacomo D. Simonetti ${ }^{4}$
}

BACKGROUND: In an attempt to improve knowledge about childhood Goodpasture's disease, we performed a retrospective analysis of patients with Goodpasture's disease from several pediatric nephrology centers.

METHODS: We analyzed the responses to 27 questions that elicited information about the following: incidence, demographics, patient history and clinical presentation, diagnostics performed, acute and chronic therapy, course of disease, and outcome.

RESULTS: Goodpasture's disease, which is extremely rare in this age group, may manifest in 2-year-old toddlers and does not typically present with pulmonary findings before puberty. Goodpasture's disease has a poor outcome with more than $50 \%$ of patients progressing to end-stage renal disease. No deaths were reported in this cohort, and renal improvement was observed in children with severe biopsy findings who required renal replacement therapy during the acute phase. CONCLUSION: The present investigation gives detailed information about childhood Goodpasture's disease under real-life conditions and reveals that very few pediatric cases have been reported. Nearly 50\% of children progressed to end-stage renal disease. However, long-term outcome in children might be better than in adults. Aggressive immunosuppressive therapy might be necessary for all affected children, even in patients who require renal replacement therapy or have severe biopsy findings.

G oodpasture's disease (GD) is a severe and extremely rare disorder in which circulating antibodies are directed against the NC1-domain of the $\alpha 3$-chain of type IV collagen, an antigen intrinsic to the glomerular basement membrane and the alveolar membrane of the lung. Affected patients typically present with both a rapidly progressive glomerulonephritis and pulmonary disease but may present with glomerulonephritis alone (1-4). GD has a poor prognosis with permanent renal function loss, which may progress to death if not treated rapidly. With adequate treatment, the prognosis may be improved; therefore, rapid recognition and treatment of GD is crucial.
There are no comprehensive data on the population incidence and prevalence of GD $(3,4)$. Very few studies of childhood GD are available $(5,6)$. In an attempt to improve knowledge about this rare condition, we performed a retrospective analysis of patients with GD from several pediatric nephrology centers.

\section{METHODS}

Between November 2013 and January 2014, we performed an international analysis on childhood GD. An invitation was sent by electronic mail to pediatric nephrologists all over the world using the international PEDNEPH mailing list, which is a mailing list for clinical questions in the field of pediatric nephrology. A link to a close-ended web-based questionnaire (hosted on www.surveymon key.com) was included, which the recipients were invited to fill in.

The cross-sectional analysis, which was not commercially sponsored, was developed by the authors and carefully tested for ease of use and clarity by different pediatric nephrologists (M.G.B. and G.A.). Cases that were previously published as case reports or case series were excluded from this analysis.

The questionnaire was written in English. Since the analysis and patient data were completely anonymous, the institutional review board waived the necessity to have approval for this type of study. In an effort to get as much information as possible about childhood GD we analyzed the responses to 27 questions that elicited information regarding the following: incidence, demographics, patient history and clinical presentation, diagnostics performed, acute and chronic therapy, course of disease, and outcome. The diagnosis of GD or anti-glomerular basement membrane antibody glomerulonephritis (anti-GBM-GN) was made if clinical, laboratory, and imaging signs of renal and/or lung involvement were present together with positive detection of antibodies against glomerular basement membrane. The full questionnaire is available as Supplementary Material.

The first three questions revealed general information about the centers, followed by 19 more specific questions. In addition, five questions regarding therapy, guidelines, and transplantation in the case of end-stage renal disease (ESRD) were developed. These latter questions could also be answered by centers that did not follow any patients with GD.

All responses remained anonymous, and no identifiers were used to identify participants in the survey.

The acute period of the disease was defined as the time at diagnosis and first therapy (usually the first 3 months from the beginning), whereas the chronic phase was 6 months from diagnosis. Acute kidney injury (AKI) refers to a sudden, potentially reversible inability of the kidney to maintain normal blood chemistry and fluid balance. Chronic kidney disease (CKD) represents an irrecoverable kidney damage lasting for at least 3 months (in this survey the long-term

\footnotetext{
${ }^{1}$ Pediatric Nephrology Unit, University Children's Hospital, Inselspital and University of Bern, Bern, Switzerland; ${ }^{2}$ Università della Svizzera Italiana, Lugano, Switzerland; ${ }^{3}$ Center for HUS Control, Prevention and Management, Fondazione IRCCS Ca' Granda Ospedale Maggiore Policlinico, Milan, Italy; ${ }^{4}$ Pediatric Department of Southern Switzerland, Bellinzona, Switzerland. Correspondence: Giacomo D. Simonetti (giacomo.simonetti@eoc.ch)

Received 8 July 2017; accepted 25 November 2017; advance online publication 10 January 2018. doi:10.1038/pr.2017.315
} 


\section{Articles | Menzi et al.}

outcome was defined as the condition after 6 months from diagnosis of GD). The condition of individuals with $\mathrm{CKD}$, who require a type of renal replacement therapy (RRT, dialysis or transplant), is referred to as the ESRD.

\section{Statistical Analysis}

Continuous data are presented as median values with interquartile range or minimum and maximum range, and categorical data are presented as frequencies and percentages calculated from single answers. Comparison of prevalence between groups was performed using the Fisher exact test. The Mann-Whitney $U$-test was used to compare two groups. Associations between individual parameters were evaluated by Spearman Rank Order correlation analysis. All statistical analyses were performed using GraphPad Prism software, version 6.0 (GraphPad Software, San Diego, CA). Significance was defined at $P<0.05$.

\section{RESULTS}

\section{Demographics}

Sixty-six pediatric nephrology centers answered the questionnaire: 27 from Europe, 17 from the USA, and two each from Asia, Australia, Canada, and South America. Fourteen centers did not mention the country. The catchment area of these centers varied between 0.02 and 25 million inhabitants (median 2.25 million). Altogether, the centers cover more than 222 million inhabitants. Twenty-eight of the sixty-six centers that replied are currently following 35 patients with

Table 1. Demographic data of 24 patients

\begin{tabular}{lc}
\hline Age at diagnosis (years) & $2-19(14)$ \\
\hline Ethnicity & $24(100 \%)$ Caucasian \\
Sex & $11(46 \%)$ female, $13(54 \%)$ male \\
Follow-up time (years) & $0.1-20(3.8)$ \\
Presentation & $18(75 \%)$ renal and pulmonary disease \\
& $6(25 \%)$ only renal disease \\
\hline $\begin{array}{l}\text { Values depicted either in absolute numbers and percentage or range with } \\
\text { median value. }\end{array}$
\end{tabular}

GD or anti-GBM-GN. Four additional centers treated five patients in the past 10 years.

\section{Detailed Data of the Patients}

More detailed information was available for 24 patients, who were treated in 17 different pediatric nephrology centers (Table 1 and Figure 1).

Patients with kidney and lung manifestations tended to be older (median 15 years, interquartile range 14-16 years) compared with children with only kidney manifestations (median 6 years, interquartile range $2.75-16$ years; $P=0.08$ ). Interestingly, no patients with both kidney and lung manifestations were diagnosed in patients under 9 years old. No relationship was found between the occurrence of combined lung and renal presentation and severity of kidney disease $(P=0.08)$ or biopsy findings $(P=0.6)$.

Nonspecific risk factors for the development of GD were found in 10 patients (smoking in 8 cases and illicit drug use in 2 cases). No hydrocarbon exposure as a known specific risk factor for the development of anti-GBM antibodies was declared. Family history of GD was not reported.

Symptoms and findings at presentation are shown in Table 2. The vast majority of patients had general symptoms and symptoms of renal impairment. Symptoms suggesting involvement of the respiratory system were found in approximately $2 / 3$ of patients.

In about $60 \%$ of the patients $(n=15)$, the time interval between the first symptoms and diagnosis of GD or antiGBM-GN was more than 1 week, and in 3 patients (12\%) the time interval was longer than 1 month.

In all cases, a renal biopsy was performed. At first presentation, more than $2 / 3$ of the patients had $>50 \%$ crescents, and $1 / 3$ had $>90 \%$ crescents. Biopsy findings were not available for two patients (Table 2 and Figure 2).

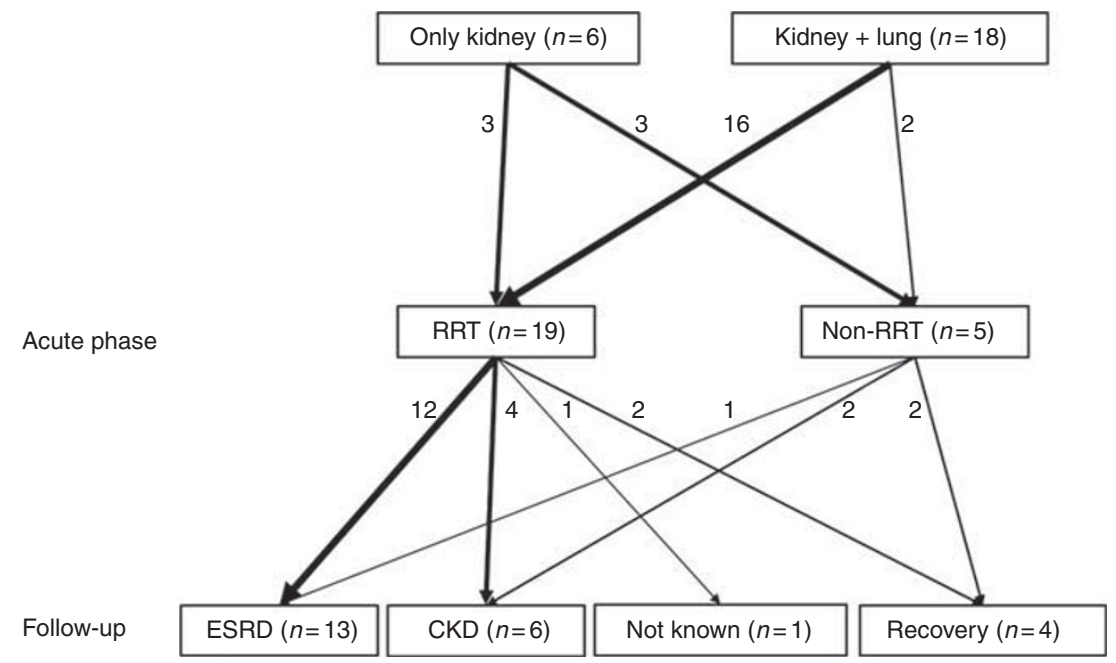

Figure 1. Flow chart describing presentation, renal function during the acute phase, and long-term renal survival. RRT, renal replacement therapy; CKD, chronic kidney disease; ESRD, end-stage renal disease. 
Table 2. Symptoms and findings at presentation, values depicted in absolute numbers and percentage

\begin{tabular}{lc}
\hline General symptoms & 18 out of $19(95 \%)$ \\
\hline Renal symptoms & 21 out of $23(91 \%)$ \\
Respiratory symptoms & 14 out of $22(63 \%)$ \\
Chest X-ray abnormalities & 16 out of $23(69 \%)$ \\
Impaired lung function & 10 out of $19(52 \%)$ \\
Renal biopsy results (crescents of glomerula) & \\
$\quad<50 \%$ & 5 out of $22(23 \%)$ \\
$50-90 \%$ & 9 out of $22(41 \%)$ \\
$\quad>90$ & 8 out of $22(36 \%)$ \\
Anti-GBM antibodies & $24(100 \%)$ \\
ANCA/ANA & $6(25 \%)$ \\
\hline
\end{tabular}

ANA, anti-nuclear antibodies; ANCA, anti-neutrophil cytoplasmatic antibodies; antiGBM antibodies, antibodies against glomerular basement membrane.

The total number of patients differ in each symptom and finding because some data were not available for all patients.

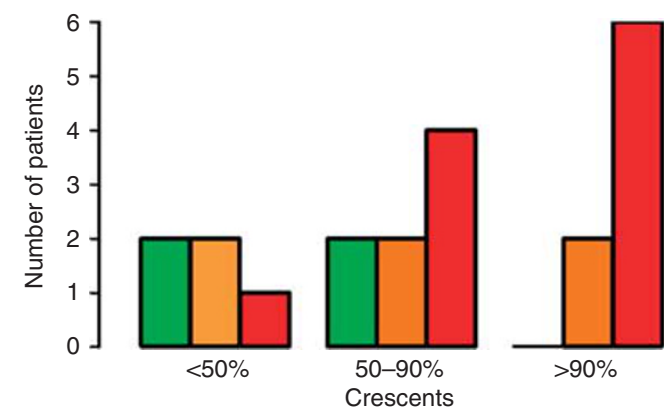

Figure 2. Long-term renal survival according to biopsy findings. Green: complete renal recovery, orange: chronic kidney disease, red: end-stage renal disease. In the group of patients with $50-90 \%$ crescents, one patient is missing because long-term renal survival was not known.

Anti-GBM antibodies were positive in all cases. Six patients also had positive ANCA (Table 2).

All patients received steroids at the time of diagnosis. The majority $(80 \%, n=19)$ also received cyclophosphamide and therapeutic plasma exchange. Mycophenolate-mofetil, azathioprine, and rituximab were each used in one patient. In this small cohort of children, no differences in therapy were found between children with kidney and lung manifestations of disease and children with only kidney manifestations. Biopsy results did not seem to influence the therapeutic choice.

The majority (almost $80 \%, n=19$ ) of patients needed RRT at the time of diagnosis, and hemodialysis $(n=17)$ was performed more commonly than peritoneal dialysis $(n=2$; Figure 1).

Four of the five patients who did not require RRT had crescents $<50 \%$ on renal biopsy. All patients with $>90 \%$ crescents on renal biopsy required RRT in the acute period.

Six months after diagnosis, the majority $(80 \%, n=19)$ were still receiving steroids. More than $50 \%(n=15)$ additionally received cyclophosphamide. Rarely, mycophenolate-mofetil and azathioprine were used in combination with steroids $(21 \%, n=5)$. Only three patients were not receiving any immunosuppressive therapy 6 months after the diagnosis of GD. These three patients all had ESRD and no lung manifestations.

Complete recovery of renal function was observed in four cases (16\%), two of which had RRT in the acute phase. Six patients (25\%) had chronic kidney disease and more than 50\% had ESRD $(n=13)$. Twelve out of these thirteen patients already had ESRD in the acute period and one patient developed ESRD during follow-up (Figure 1). The relationship between long-term renal survival and renal biopsy findings is depicted in Figure 2 and Table 3. The figure shows that more crescents tend to be associated with a poorer prognosis $(P=0.02)$.

Outcome was not influenced by the patient's age at diagnosis. Initial interval in diagnosis was not associated with the severity of biopsy findings, need for RRT, or long-term outcome (Table 3). Outcomes for symptoms of respiratory disease were good, with complete recovery in $>80 \%$ of the patients, with only three patients having some residual pulmonary findings on long-term follow-up. No deaths occurred in this cohort of patients.

\section{General Questions About Management of Goodpasture's Disease in Childhood}

Conventional chest X-ray was performed in all patients, with or without respiratory symptoms, whereas CT scan or MRI were only performed in $1 / 3$ of the centers if respiratory symptoms were present. Similarly, bronchoalveolar lavage or lung function testing were always performed in 14\% and $42 \%$ of the centers, respectively; if respiratory symptoms were present, these tests were performed in $62 \%$ and $49 \%$ of the centers, respectively.

KDIGO adult guidelines (7) recommend avoiding immunosuppressive treatment in patients who are dependent on dialysis at presentation or who have $100 \%$ crescents in an adequate biopsy sample, in the absence of pulmonary hemorrhage, because the chance of recovery of renal function in these situations is very low (near $0 \%$ in many studies) (810). Forty-five percent of the centers surveyed also follow this guideline for children (only $27 \%$ of these centers had already treated children with GD), whereas $55 \%$ of the centers do not follow these recommendations ( $45 \%$ of these centers had already treated children with GD).

The majority of the centers (58\%) wait at least 12 months after anti-GBM antibodies are no longer detectable without immunosuppressive therapy before performing kidney transplantation. Twenty-five percent wait at least 6 months and the other centers wait at least 18 months. Almost all the centers also perform a living donor transplantation.

\section{DISCUSSION}

This study aims to improve knowledge of the clinical presentation, management, and outcomes of childhood GD. 


\section{Articles | Menzi et al.}

Table 3. Correlation analysis (Spearman rank-order correlation analysis)

\begin{tabular}{|c|c|c|c|c|}
\hline & Initial interval & Biopsy (crescents) & Need for RRT during acute phase & Long-term outcome \\
\hline \multirow[t]{2}{*}{ Age at diagnosis (years) } & $r=-0.13$ & $r=-0.09$ & $r=-0.11$ & $r=-0.01$ \\
\hline & $P=0.58$ & $P=0.67$ & $P=0.60$ & $P=0.96$ \\
\hline \multirow[t]{2}{*}{ Initial interval } & & $r=0.13$ & $r=0.19$ & $r=-0.05$ \\
\hline & & $P=0.57$ & $P=0.39$ & $P=0.81$ \\
\hline \multirow[t]{2}{*}{ Biopsy (crescents) } & & & $r=0.65$ & $r=0.50$ \\
\hline & & & $P=\mathbf{0 . 0 0 1}$ & $P=\mathbf{0 . 0 2}$ \\
\hline Need for RRT & & & & $r=0.41$ \\
\hline during acute phase & & & & $P=\mathbf{0 . 0 5}$ \\
\hline
\end{tabular}

RRT, renal replacement therapy; $r$, correlation coefficient.

Bold values are statistically significant correlations.

The main results reveal that the disease, which is extremely rare in this age group, may manifest in toddlers, and does not present commonly with pulmonary findings before puberty. This latter finding is interesting and has not been previously described. A possible pathophysiological explanation is that the structure of the lung basement membrane in childhood is without anti-GBM antigen presentation, as has been previously suggested $(5,11)$.

Another interesting finding in this analysis is that $1 / 4$ of the pediatric patients with GD also had nonspecifically increased anticytoplasmatic antibodies (ANCA). In adults, the presence of ANCA is described in $\sim 20-30 \%$ of patients, whereas in children this phenomenon has been described in $17 \%$ of patients $(5,12)$. The results also confirm that GD has poor outcomes, with $50 \%$ of the affected children developing ESRD. Poor outcome is associated with biopsy findings and need for RRT during the acute phase. Nevertheless, these data suggest that long-term outcome in children might be better than in adults; no deaths were reported and renal improvement was observed even in children with severe biopsy findings ( $>90 \%$ crescents) who required RRT during the acute phase. These findings are in contrast to a previous systematic review of GD in children (5), which described a case fatality rate similar to adults. These results are also in contrast to adult guidelines, which recommend avoiding immunosuppressive treatment in patients who require dialysis at first presentation and who have $100 \%$ crescents in an adequate biopsy sample, in the absence of pulmonary hemorrhage (7). It seems, therefore, that adult guidelines should not be applied to children and that childhood-specific guidelines are warranted. A possible explanation of this difference is the increased plasticity and potential for regeneration of the renal tissue of children. In this context, it is important to differentiate between cellular and fibrous crescents on biopsy findings. The former have increased chances of regeneration, whereas the latter represent scar tissue, which is non-regenerative. These results suggest that aggressive immunosuppressive therapy should also be started immediately in patients without pulmonary involvement who need RRT and have severe biopsy findings with cellular crescents. Interestingly, centers with clinical experience with this disease more frequently do not adhere to adult guidelines, probably indicating that in rare disease such as GD reference centers for proper management are warranted. The standard therapy consists of corticosteroids, cyclophosphamide, and plasmapheresis. Before kidney transplantation, at least 1 year without the presence of antibodies is typically required. The results also clearly show that more than half of the patients had a time interval of more than 1 week between the development of the first symptoms and diagnosis. Although this time interval was not associated with a worse outcome, this finding emphasizes the need to improve awareness of GD among pediatricians.

The main results are similar to those of the systematic review performed by Bayat et al. (5). The differences noted include differences in mortality rate, as stated above, and sex distribution (in the present cohort males and females were equally distributed).

A positive aspect of this analysis compared with the previous review performed by Bayat et al. (5) is that it represents a study in the field of pediatric nephrology under real-life conditions and not a systematic review of the literature with the possibility of publication bias.

However, there were some limitations to this study. First, the low number of cases of childhood GD do not allow for a valid statistical analysis and interpretation of the results needs caution. Nevertheless, this limitation is often encountered when studying rare diseases. Second, due to the unavoidable retrospective nature of this analysis, some of the reported cases were not described in detail; therefore, some data were missing or inaccurate, with a potential for recollection bias. Third, the majority of the centers surveyed were from the United States and Europe and therefore not representative for the total population. Moreover, this issue represents an obvious selection bias because most of these centers are from developed or relatively developed countries, where highquality medical service may be available. Finally, we cannot exclude the possibility of underreporting of childhood cases of GD by the involved centers or the potential of missed undiagnosed cases of GD in childhood.

In conclusion, the present analysis gives detailed information regarding the clinical presentation, management, and outcomes of childhood GD under real-life conditions, reiterating that this disease is extremely rare. In childhood, 
despite aggressive management, nearly $50 \%$ of children developed ESRD. The most common treatment strategies for childhood GD includes immediate aggressive immunosuppressive therapy, even in patients who require RRT or who have severe biopsy findings. Standard therapy includes corticosteroids, cyclophosphamide, and plasmapheresis.

\section{SUPPLEMENTARY MATERIAL}

Supplementary material is linked to the online version of the paper at http://www.nature.com/pr

\section{ACKNOWLEDGMENTS}

We acknowledge Fondazione E. Balli for the support.

Disclosure: The authors declare no conflict of interest.

\section{REFERENCES}

1. Goodpasture EW. The significance of certain pulmonary lesions in relation to the etiology of influenza. Am J Med Sci 1919;158:863-70.

2. Stanton MC, Tange JD. Goodpasture's syndrome (pulmonary haemorrhage associated with glomerulonephritis). Australas Ann Med 1958;7:132-44

3. Greco A, Rizzo MI, De Virgilio A, et al. Goodpasture's syndrome: a clinical update. Autoimmun Rev 2015;14:246-53.
4. Hudson BG, Tryggvason K, Sundaramoorthy M, Neilson EG. Alport's syndrome, Goodpasture's syndrome, and type IV collagen. N Engl J Med 2003;348:2543-56.

5. Bayat A, Kamperis K, Herlin T. Characteristics and outcome of Goodpasture's disease in children. Clin Rheumatol 2012;31:1745-51.

6. Williamson SR, Phillips CL, Andreoli SP, Nailescu C. A 25-year experience with pediatric anti-glomerular basement membrane disease. Pediatr Nephrol 2011;26:85-91.

7. [No authors listed]. Chapter 14: anti-glomerular basement membrane antibody glomerulonephritis. Kidney Int Suppl 2012;2:240-2.

8. Levy JB, Hammad T, Coulthart A, Dougan T, Pusey CD. Clinical features and outcome of patients with both ANCA and anti-GBM antibodies. Kidney Int 2004;66:1535-40.

9. Segelmark M, Hellmark T, Wieslander J. The prognostic significance in Goodpasture's disease of specificity, titre and affinity of anti-glomerularbasement-membrane antibodies. Nephron Clin Pract 2003;94:c59-68.

10. Levy JB, Turner AN, Rees AJ, Pusey CD. Long-term outcome of anti-glomerular basement membrane antibody disease treated with plasma exchange and immunosuppression. Ann Intern Med 2001;134: 1033-42.

11. Anand SK, Landing BH, Heuser ET, Olson DL, Grushkin CM, Lieberman E. Changes in glomerular basement membrane antigen(s) with age. J Pediatr 1978;92:952-3.

12. Naidoo S, Waller S. Anti-GBM antibodies co-exist with MPO-ANCA in a 4-year-old girl with acute renal failure. Pediatr Nephrol 2009;24:215-6. 